\title{
KRAS Gene Mutations and Gender Differences in Colorectal Cancer
}

\author{
Oleg I. Kit, PhD, ScD; Dmitriy I. Vodolazhskiy, PhD; Yuriy A. Gevorkyan, PhD, ScD; \\ Natalia V. Soldatkina, $\mathrm{PhD}, \mathrm{ScD}^{*}$ \\ Federal State Budget Institution «Rostov Research Oncologic Institute» \\ Rostov-on-Don, the Russian Federation
}

\begin{abstract}
The aim of this study was to investigate the frequency and spectrum of KRAS mutations in men and women with colorectal cancer (CRC), and an impact of KRAS-mutation status on the clinical and morphological features of CRC. The study included 303 patients (168/55.4\% women and 135/44.6\% men) with CRC T2-4N0-2M0-1. We defined 7 KRAS SNP-mutations (G12D, G12A, G12R, G12C, G12S, G12V and G13D) located within codons 12 and 13 using Bio-Rad real-time thermal cyclers CFX96 and Real-Time-PCR- KRAS-7M Kit. The frequency of KRAS mutations was $35.6 \%$ in the CRC patients with a predominant presence of $\mathrm{G}>\mathrm{A}$ transitions. The KRAS codon 12 and 13 mutations are predictive of poor prognosis The KRAS-mutated CRC has clinical features in view of the gender differences. KRAS-mutation status is a promising predictive biomarker of personalized treatment.
\end{abstract}

Keywords: KRAS codon 12 and 13 mutations; colorectal cancer; gender differences.

\section{Introduction}

Colorectal cancer (CRC) is one of the most common cancers worldwide [1]. This localization of a malignant tumor is a prime example of the successful application of the fundamental advances in clinical practice [2]. In the last decade, significant improvements have been made in response rates, progression-free survival, and overall survival [3-6]. These significant improvements are mainly a result of the development of new combinations of standard chemotherapy and also new therapeutic agents targeting molecular events involved in colorectal carcinogenesis.

One of the most promising targets is the epidermal growth factor receptor (EGFR), which is activated in colorectal carcinogenesis by the binding of a ligand on the extracellular part of it [7]. With the emergence of two antieEGFR-targeted antibodies, cetuximab and panitumumab, the treatment of metastatic colorectal cancer has entered into the era of personalized treatment. However, EGFR, the target of these drugs, which is over expressed in approximately $80 \%$ of colorectal carcinomas, failed to predict a therapeutic response when used clinically $[8,9]$. Therefore, downstream signaling effectors were sought to help predict the efficacy of

*Corresponding author: Natalia V. Soldatkina. PhD, ScD. Rostov Research Oncologic Institute. Rostov-on-Don, the Russian Federation.E-mail:snv-rnioi@yandex.ru
anti-EGFR treatment.

KRAS is a small G protein that acts as a transducer in the EGFR pathway. KRAS is a membrane-anchored guanosine triphosphate/guanosine diphosphate (GTP/GDP)-binding protein and is widely expressed in most human cells. As a small GTPase (GTP cleaving enzyme), KRAS is involved in intracellular signal transduction and mainly responsible for EGFR-signaling activation [10]. The exchange of the active GTP-bound state and the inactive GDP-bound state is tightly controlled by GTPase-activating proteins (GAPs) and guanine nucleotide exchange factors [11]. Under normal physiological conditions, upstream signals activate wild-type KRAS by promoting the exchange of bound GDP for GTP. This process is transient because of GAP-mediated GTP hydrolysis. However, this process becomes altered when the KRAS gene is mutated. When there is a point mutation in codon 12 [12], the protein is locked in the active state and constitutively transmits to the nucleus mitogenic signals.

Mutant KRAS is found in about $35 \%-45 \%$ of CRCs [1315], and codon 12 and 13 are two hotspots, which account for about $95 \%$ of all mutation types, with approximately $80 \%$ occurring in codon 12 and $15 \%$ in codon 13 . KRAS mutations are almost single nucleotide point mutations as reported, and the most common patterns are G12D, G12A, G12R, G12C, G12S, G12V and G13D. In the codon 12 mutation, p.G12D, pG12V is the most frequent, and in codon 13, the substitution of glycine for aspartate (p.G13D) is the most frequent [16]. 
These mutations impair the intrinsic GTPase activity of KRAS and prevent GAPs from promoting GTP hydrolysis by KRAS, therefore causing KRAS proteins to accumulate in the GTP-bound, active form. In this manner, mutant KRAS results in a constitutively active GTP-bound state and the activation of downstream pro-proliferative signaling pathways $[17,18]$. Therefore, KRAS mutations play a critical role in human tumorigenesis and are the most prevalent in colorectal cancer. Because $K R A S$ is the most frequently mutated factor downstream of the EGFR signaling pathway, it was considered a candidate molecular biomarker for anti-EGFR therapy [19]. At the same time the associations between KRASmutation status and the clinical, morphological, and biological characteristics of $\mathrm{CRC}$, as well as gender differences, are unclear [20,21].

The aim of this study was to investigate the frequency and spectrum of KRAS mutations in men and women with $\mathrm{CRC}$, and an impact of KRAS-mutation status on the clinical and morphological features of CRC.

\section{Material and Methods}

The study included 303 patients $(168 / 55.4 \%$ women and $135 / 44.6 \%$ men) with CRC T2-4N0-2M0-1 treated in the Rostov Cancer Research Institute between 2011 and 2014. The age distribution was as follows: the age group under 45 years included 26(8.6\%) patients, the 45-to-54 age group included $54(17.8 \%)$ patients, the 55-to-64 age group 131(43.2\%) patients, and age group over 65 years included 92(30.4\%) patients.

The tumor was located in the ampullar part and anal canal of the rectum in $127(41.9 \%)$ patients, in the rectosigmoid part of the rectum in $97(32.0 \%)$ patients, in the left side of the descending colon in $30(9.9 \%)$ patients, and in the right side of the descending colon in 49(16.2\%) patients. In all patients, histologically, the tumor was characterized as an adenocarcinoma of varying degrees of differentiation (G2 in 266/87.8\% patients, G3 in 29/9.6\% patients, and G1 in $8 / 2.6 \%$ patients). We revealed the following T-stages of CRC: T4-stage in $158(52.1 \%)$ patients, T3-stage in 80(26.4\%), T2stage in $63(20.8 \%)$, and T1-stage in $2(0.7 \%)$ patients. All the patients underwent the cytoreductive and radical surgery.

DNA was extracted from paraffin-embedded, formalinfixed tumor tissue using a QIAamp ${ }^{\circledR}$ DNA FFPE Tissue Kit (QIAGENE, Germany) according to the manufacturer's protocol. The concentration of the extracted DNA was measured by a Qubit 2.0® Fluorometer using Quant-iT TM dsDNA Kit. The concentration of DNA was normalized to a value of $1 \mathrm{ng} / \mathrm{ml}$.

We defined 7 KRAS SNP-mutations (G12D, G12A, G12R, G12C, G12S, G12V and G13D) located within codons 12 and 13 using Bio-Rad real-time thermal cyclers CFX96 and Real-Time-PCR- KRAS-7M Kit.

Results were statistically processed using the software package Statistica 8.0 and the Excel package of Microsoft Excel 2010. We used the Chi-square test to compare observed data. A probability value of $\mathrm{P}<0.05$ was considered statistically significant.

\section{Results and Discussion}

KRAS codon 12 and 13 mutations were detected in $108(35.6 \%)$ patients, which is consistent with other studies [20]. KRAS-mutated CRC was identified in 65(60.2\%) women and in 43(39.8\%) men (Table 1).

Table 1.

Clinical and morphological features of CRC in patients with KRAS codon 12 and 13 mutations

\begin{tabular}{|l|c|c|}
\hline \multicolumn{1}{|c|}{ Features } & Women $(\mathrm{n}=65)$ & Men $(\mathrm{n}=43)$ \\
\hline Age distribution & & \\
under 45 years & $10(15.3 \%)$ & $1(2.3 \%)^{*}$ \\
45-to-54 years & $5(7.7 \%)$ & $3(7.0 \%)$ \\
55-to-64 years & $25(38.5 \%)$ & $23(53.5 \%)^{*}$ \\
over 65 years & $25(38.5 \%)$ & $16(37.2 \%)$ \\
\hline Localization of tumor & & \\
rectum & $31(47.7 \%)$ & $14(32.6 \%)^{*}$ \\
rectosigmoid area & $18(27.7 \%)$ & $14(32.6 \%)$ \\
the left side of the DC & $8(12.3 \%)$ & $6(14.0 \%)$ \\
the right side of the DC & $8(12.3 \%)$ & $9(20.8 \%)$ \\
\hline Histology: & $2(3.0 \%)$ & \\
G1 adenocarcinoma & $56(86.2 \%)$ & $40(93.0 \%)$ \\
G2 adenocarcinoma & $7(10.8 \%)$ & $3(7.0 \%)$ \\
G3 adenocarcinoma & & \\
\hline T-stage & $11(16.9 \%)$ & $2(4.7 \%)$ \\
2 & $18(27.7 \%)$ & $12(27.9 \%)$ \\
3 & $36(55.4 \%)$ & $28(65.1 \%)^{*}$ \\
4 & & \\
KRAS mutation & $40(61.5 \%)$ & $27(62.8 \%)$ \\
G $>$ A transitions & $13(20.0 \%)$ & $8(18.6 \%)$ \\
G $>$ T transversions & $12(18.5 \%)$ & $8(18.6 \%)$ \\
G>C transversions & & \\
\hline
\end{tabular}

Note: $D C$ - descending colon; ${ }^{*}-P<0.05$ between groups.

Among patients with KRAS-mutated CRC, the age distribution was as follows: the age group under 45 years included 11(10.2\%) patients, the 45-to-54 age group included $8(7.4 \%)$ patients, the 55-to-64 age group $48(44.4 \%)$ patients, and age group over 65 years included $41(38.0 \%)$ patients. The tumor was located in the ampullar part and anal canal of the rectum in $45(41.7 \%)$ patients, in the rectosigmoid part of the rectum in 32(29.6\%) patients, in the left side of the descending colon in $14(13.0 \%)$ patients, and in the right side of the descending colon in $17(15.7 \%)$ patients. The postoperative histological study found that a moderately differentiated adenocarcinoma was predominant in patients $(96 / 88.9 \%)$ with KRAS-mutated CRC. Poorly differentiated adenocarcinoma was found in $10(9.3 \%)$ patients and well differentiated adenocarcinoma only in $2(1.8 \%)$ patients. In patients with KRAS-mutated CRC, we revealed the following T-stages of CRC: T4-stage in 64(59.3\%) patients, T3-stage in 30(27.8\%), T2-stage in $13(12.0 \%)$, and T1-stage in $1(0.9 \%)$ patients.

$\mathrm{G}>\mathrm{A}$ transitions (G12S, G12D, and G13D mutations) were detected in $67(62.0 \%)$ patients. This type of mutation was found in $40(59.7 \%)$ women and $27(40.3 \%)$ men. The age distribution was as follows: the age group under 45 years included $7(10.4 \%)$ patients, the 45 -to-54 age group included $6(8.9 \%)$ patients, the 55-to-64 age group 31(46.3\%) patients, 
and age group over 65 years included 23(34.4\%) patients. The tumor was located in the ampullar part and anal canal of the rectum in $28(41.8 \%)$ patients, in the rectosigmoid part of the rectum in $15(22.4 \%)$ patients, in the left side of the descending colon in $9(13.4 \%)$ patients, and in the right side of the descending colon in $15(22.4 \%)$ patients. Histologically, G>A transitions were associated with a moderately differentiated adenocarcinoma in 56(83.6\%) cases. Poorly differentiated adenocarcinoma was found in $9(13.4 \%)$ patients and well differentiated adenocarcinoma only in $2(3.0 \%)$ patients. In patients with $\mathrm{G}>\mathrm{A}$ transitions, we revealed the following T-stages of CRC: T4-stage in 37(55.2\%) patients, T3-stage in $20(29.9 \%)$, and T2-stage in $10(14.9 \%)$.

$\mathrm{G}>\mathrm{T}$ transversions (G12V and G12C mutations) were detected in $21(19.4 \%)$ patients. This type of mutation was found in $13(61.9 \%)$ women and $8(38.1 \%)$ men. The age distribution was as follows: the age group under 45 years included $2(9.5 \%)$ patients, the 45 -to-54 age group included $2(9.5 \%)$ patients, the 55-to-64 age group $8(38.1 \%)$ patients, and age group over 65 years included $9(42.9 \%)$ patients. The tumor was located in the ampullar part and anal canal of the rectum in $6(28.6 \%)$ patients, in the rectosigmoid part of the rectum in $10(47.6 \%)$ patients, in the left side of the descending colon in $4(19.0 \%)$ patients, and in the right side of the descending colon in 1(4.8\%) patients. Histologically, $\mathrm{G}>\mathrm{T}$ transversions were associated with a moderately differentiated adenocarcinoma in 20(95.2\%) cases. Poorly differentiated adenocarcinoma was found in 1(4.8\%) patients; well differentiated adenocarcinoma was not found. In patients with $\mathrm{G}>\mathrm{T}$ transversions, we revealed the following T-stages of CRC: T4-stage in $16(76.2 \%)$ patients, T3-stage in 4(19.0\%), and T2-stage in $1(4.8 \%)$.

$\mathrm{G}>\mathrm{C}$ transversions (G12A and G12R mutation) were detected in $20(18.6 \%)$ patients. This type of mutation was found in $12(60.0 \%)$ women and $8(40.0 \%)$ men. The age distribution was as follows: the age group under 45 years included 2(10.0\%) patients, the 55-to-64 age group 9(45.5\%) patients, and age group over 65 years included 9(45.5\%) patients. The tumor was located in the ampullar part and anal canal of the rectum in $11(55.0 \%)$ patients, in the rectosigmoid part of the rectum in $7(35.0 \%)$ patients, in the left side of the descending colon in $1(5.0 \%)$ patients, and in the right side of the descending colon in $1(5.0 \%)$ patients. Histologically, $\mathrm{G}>\mathrm{C}$ transversions were associated with a moderately differentiated adenocarcinoma in $19(95.0 \%)$ cases. Poorly differentiated adenocarcinoma was found in $1(5.0 \%)$ patients; well differentiated adenocarcinoma was not found. In patients with $\mathrm{G}>\mathrm{C}$ transversions, we revealed the following T-stages of CRC: T4-stage in $12(60.0 \%)$ patients, T3-stage in 6(30.0\%), T2-stage in 2(10.0\%).

KRAS codon 12 and 13 mutations were not detected in 195(64.4\%) patient. KRAS-wild-type CRC was found in 103(52.8\%) women and 92(47.2\%) men. In patients with KRAS-wild-type CRC, the age distribution was as follows: the age group under 45 years included $15(7.7 \%)$ patients, the 45-to-54 age group included 46(23.6\%) patients, the 55to-64 age group 83(42.6\%) patients, and age group over 65 years included 51(26.1\%) patients. The tumor was located in the ampullar part and anal canal of the rectum in $82(42.1 \%)$ patients, in the rectosigmoid part of the rectum in $65(33.3 \%)$ patients, in the left side of the descending colon in 16(8.2\%) patients, and in the right side of the descending colon in $32(16.4 \%)$ patients. Histologically, KRAS-wild-type CRC was associated with a moderately differentiated adenocarcinoma in $170(87.2 \%)$ cases. Poorly differentiated adenocarcinoma was found in $19(9.7 \%)$ patients, well differentiated adenocarcinoma in 6(3.1\%) patients. In patients with KRASwild-type CRC, we revealed the following T-stages of CRC: T4-stage in $99(50.8 \%)$ patients, T3-stage in $45(23.1 \%)$, T2stage in 50(25.7\%), and T1-stage in 1(0.4\%).

Results of the analysis of clinical and morphological features of CRC based on gender differences are shown in Tables 2 and 3.

Table 2.

Clinical and morphological features of CRC in women with KRAS codon 12 and 13 mutations

\begin{tabular}{|l|c|c|c|}
\hline \multicolumn{1}{|c|}{ Features } & $\begin{array}{c}\mathrm{G}>\mathrm{A} \\
\text { transitions } \\
(\mathrm{n}=40)\end{array}$ & $\begin{array}{c}\mathrm{G}>\mathrm{T} \\
\text { transversions } \\
(\mathrm{n}=13)\end{array}$ & $\begin{array}{c}\mathrm{G}>\mathrm{C} \\
\text { transversions } \\
(\mathrm{n}=12)\end{array}$ \\
\hline Age distribution & $6(15.0 \%)$ & $2(15.4 \%)$ & $2(6.6 \%)$ \\
under 45 years & $3(7.5 \%)$ & $2(15.4 \%)$ & - \\
45-to-54 years & $15(37.5 \%)$ & $5(38.5 \%)$ & $5(41.7 \%)$ \\
$55-$-to-64 years & $16(40.0 \%)$ & $4(30.7 \%)$ & $5(41.7 \%)$ \\
over 65 years & $19(47.5 \%$ & $5(38.5 \%)$ & $6(50.0 \%)$ \\
\hline Localization of tumor & $7(17.5 \%)$ & $6(46.1 \%)$ & $6(50.0 \%)$ \\
rectum & $6(15.0 \%)$ & $2(15.4 \%)$ & - \\
rectosigmoid area & $8(20.0 \%)$ & - & - \\
the left side of the DC \\
the right side of the DC
\end{tabular}

Among the 168 women with CRC, KRAS codon 12 and 13 mutations were detected in 65(38.7\%) patients. Most women with KRAS-mutated CRC were over 55 years of age. Among the KRAS codon 12 and 13 mutations in the CRC women, $\mathrm{G}>\mathrm{A}$ transitions were predominated $(40 / 61.5 \%$ of patients). $\mathrm{G}>\mathrm{T}$ and $\mathrm{G}>\mathrm{C}$ transversions were detected in $13(20.0 \%)$ and $12(18.5 \%)$ women, respectively. Among the 168 CRC women, the KRAS-wild-type CRCs were observed in $103(61.3 \%)$ patients.

Among the 135 men with CRC, KRAS codon 12 and 13 mutations were detected in 43(31.9\%) patients. Most men with KRAS-mutated CRC were over 55 years of age. Among the KRAS codon 12 and 13 mutations in the CRC men, $\mathrm{G}>\mathrm{A}$ transitions were predominated (27/62.8\% of patients). $\mathrm{G}>\mathrm{T}$ and $\mathrm{G}>\mathrm{C}$ transversions were detected in $8(18.6 \%)$ and $8(18.6 \%)$ men, respectively. Among the $168 \mathrm{CRC}$ women, the KRAS-wild-type CRCs were observed in 92(68.1\%) patients. 
Table 3.

Clinical and morphological features of CRC in men with KRAS codon 12 and 13 mutations

\begin{tabular}{|c|c|c|c|}
\hline Features & $\begin{array}{l}\mathrm{G}>\mathrm{A} \\
\text { transitions } \\
(\mathrm{n}=27)\end{array}$ & $\begin{array}{c}\mathrm{G}>\mathrm{T} \\
\text { transversions } \\
(\mathrm{n}=8)\end{array}$ & $\begin{array}{c}\mathrm{G}>\mathrm{C} \\
\text { transversions } \\
(\mathrm{n}=8)\end{array}$ \\
\hline $\begin{array}{l}\text { Age distribution } \\
\text { under } 45 \text { years } \\
45 \text {-to-54 years } \\
55 \text {-to- } 64 \text { years } \\
\text { over } 65 \text { years }\end{array}$ & $\begin{array}{c}1(3.7 \%) \\
3(11.1 \%) \\
16(59.3 \%) \\
7(25.9 \%)\end{array}$ & $\begin{array}{c}- \\
- \\
3(37.5 \%) \\
5(62.5 \%)\end{array}$ & $\begin{array}{c}- \\
- \\
4(50.0 \%) \\
4(50.0 \%)\end{array}$ \\
\hline $\begin{array}{l}\text { Localization of tumor } \\
\text { rectum } \\
\text { rectosigmoid area } \\
\text { the left side of the DC } \\
\text { the right side of the DC }\end{array}$ & $\begin{array}{l}9(33.3 \%) \\
8(29.7 \%) \\
3(11.1 \%) \\
7(25.9 \%)\end{array}$ & $\begin{array}{l}1(12.5 \%) \\
4(50.0 \%) \\
2(25.0 \%) \\
1(12.5 \%)\end{array}$ & $\begin{array}{l}5(62.5 \%) \\
1(12.5 \%) \\
1(12.5 \%) \\
1(12.5 \%)\end{array}$ \\
\hline $\begin{array}{l}\text { Histology: } \\
\text { G1 adenocarcinoma } \\
\text { G2 adenocarcinoma } \\
\text { G3 adenocarcinoma }\end{array}$ & $\begin{array}{c}- \\
25(92.6 \%) \\
2(7.4 \%)\end{array}$ & $\begin{array}{c}- \\
7(87.5 \%) \\
1(12.5 \%)\end{array}$ & $\begin{array}{c}- \\
7(87.5 \%) \\
1(12.5 \%)\end{array}$ \\
\hline \begin{tabular}{|l} 
T-stage \\
2 \\
3 \\
4
\end{tabular} & $\begin{array}{c}1(3.7 \%) \\
8(29.7 \%) \\
18(66.6 \%)\end{array}$ & $\begin{array}{l}1(12.5 \%) \\
1(12.5 \%) \\
6(75.0 \%)\end{array}$ & $\begin{array}{c}- \\
3(37.5 \%) \\
5(62.5 \%)\end{array}$ \\
\hline
\end{tabular}

Our study showed no difference in the frequency of the KRAS codon 12 and 13 mutations in men (43/31.9\%) and women (65/38.7\%) with CRC. KRAS gene mutations were more common when a tumor was located in the rectum $(P<0.05)$. KRAS-mutated CRC was presented as a moderately differentiated adenocarcinoma in $96 / 88.9 \%$ of patients $(P<0.05)$ and characterized by a high degree of tumor spread. After surgery, T3-4 states were significantly predominant in the KRAS-mutated CRC than the KRAS-wild-type CRC $(87.1 \%$ vs. $73.9 \%, P<0.05)$. These data indicate a worsening prognosis in the CRC patients with KRAS codon 12 and 13 mutations. In our study, among the KRAS codon 12 and 13 mutations, $\mathrm{G}>\mathrm{A}$ transitions were predominant (66.2\%). We revealed some patterns of clinical manifestations depending on the mutation type. G>A transitions compared to other mutations were associated with tumor localization in the right side of the descending colon in a larger number of cases $(P<0.05) . \mathrm{G}>\mathrm{T}$ transversions, compared to other mutations, were associated with tumor localization in the ampullar part in a larger number of cases and in the anal canal of rectum in fewer cases $(P<0.05)$. In contrast, $\mathrm{G}>\mathrm{C}$ transversions were associated with a tumor localization in the rectum in $90 \%$ of cases $(P<0.05)$. G>T transversions compared to other mutations were associated with T4-stage of CRC with the highest frequency $(76.2 \%, P<0.05)$.

Women with KRAS-mutated CRC compared to men were more often in the age group under 45 years (15.3 and $2.3 \%$, respectively) and less in the 55- to- 64 year age group (38.5 and $53.5 \%$, respectively, $P<0.05$ ). In women in the age group over 65 years compared to men, $\mathrm{G}>\mathrm{A}$ transitions were more common ( $40 \%$ and $25.9 \%$, respectively); on the contrary, $\mathrm{G}>\mathrm{T}$ transversions were more rare $(30.7$ and $62.5 \%$, respectively, $P<0.05$ ). In women with KRAS-mutated CRC compared to men, tumor was often localized in the ampullar part and the anal canal of the rectum $(47.7 \%$ and $32.6 \%$, respectively, $P<0.05$ ) that was associated with an increase in the number of $\mathrm{G}>\mathrm{A}$ transitions and $\mathrm{G}>\mathrm{T}$ transversions. In women with $\mathrm{G}>\mathrm{C}$ transversions compared to men, tumor was detected only in the rectum ( $100 \%$ vs $75 \%$ patients, $P<0.05)$. $\mathrm{G}>\mathrm{T}$ and $\mathrm{G}>\mathrm{C}$ transversions in men compared to women were more often associated with the localization of tumor in the descending colon ( 37.5 and $25.0 \%$ vs. $15.4 \%$ and $0 \%$, respectively, $P<0.05)$. In men with KRAS-mutated CRC, T4stage of CRC was diagnosed significantly more often in the background of the high frequency of $\mathrm{G}>\mathrm{A}$ transitions.

Thus, the KRAS codon 12 and 13 mutations are more common in tumors with the rectum localization and T3-4 stages of the disease. These mutations are predictive of poor prognosis. Identified patterns of CRC clinical manifestations according to gender and type of mutations allow to determine the potential biological behavior of CRC based on the assessment of the KRAS-mutation status and create the possibility for personalized approach to therapy and optimize an individual monitoring of patient condition.

\section{Conclusions}

- The frequency of KRAS mutations was $35.6 \%$ in the CRC patients with a predominant presence of $\mathrm{G}>\mathrm{A}$ transitions (62\%). The KRAS codon 12 and 13 mutations were associated with the tumor localization in the rectum $(71.3 \%)$ and a moderately differentiated adenocarcinoma $(88.9 \%, P<0.05)$.

- The KRAS codon 12 and 13 mutations are predictive of poor prognosis. The presence of these mutations compared to KRAS-wild-type CRC was significantly more associated with T3-4 stages of CRC.

- The KRAS-mutated CRC has clinical features in view of the gender differences. Women with KRAS-mutated CRC compared to men were more often in the age group under 45 years $(15.3$ vs. $2.3 \%, P<0.05)$ and less in the 55 -to-64 year age group ( 38.5 vs. $53.5 \%, P<0.05)$. In women with $\mathrm{G}>\mathrm{C}$ transversions compared to men, tumor was detected only in the rectum ( $100 \%$ vs $75 \%$ patients, $P<0.05)$. G $>\mathrm{T}$ and $\mathrm{G}>\mathrm{C}$ transversions in men compared to women were more often associated with the localization of tumor in the descending colon $(37.5$ and $25.0 \%$ vs. $15.4 \%$ and $0 \%$, respectively, $P<0.05$ ). In men with KRAS-mutated CRC, T4-stage of CRC was diagnosed significantly more often in the background of the high frequency of $\mathrm{G}>\mathrm{A}$ transitions.

\section{Competing interests}

The authors declare that they have no competing interests.

\section{References}

1. Chissov VI, Starinskiy VV, Petrova GV. Malignancies in Russia in 2011 (morbidity and mortality). M.: FSBI "MORI n.a. P.A. Herzen" of Ministry of Healthcare of RF; 2013.

2. Kit OI. The problem of colorectal cancer in the early twenty-first century: achievements and perspectives. Rus J Gastroenterol Hepatol Coloproctol 2013; 3(23):65-71. 
3. de Gramont A, Figer A, Seymour M, Homerin M, Hmissi A, Cassidy J, et al. Leucovorin and fluorouracil with or without oxaliplatin as first-line treatment in advanced colorectal cancer. J Clin Oncol 2000; 18(16):2938-47.

4. Douillard JY, Cunningham D, Roth AD, Navarro M, James $\mathrm{RD}$, Karasek P, et al. Irinotecan combined with fluorouracil compared with fluorouracil alone as first-line treatment for metastatic colorectal cancer: a multicentre randomized trial. Lancet 2000; 355(9209):1041-7.

5. Goldberg RM, Sargent DJ, Morton RF, Fuchs CS, Ramanathan RK, Williamson SK, et al. A randomized controlled trial of fluorouracil plus leucovorin, irinotecan, and oxaliplatin combinations in patients with previously untreated metastatic colorectal cancer. J Clin Oncol 2004; 22(1):23-30.

6. Saltz LB, Clarke S, Díaz-Rubio E, Scheithauer W, Figer A, Wong R, et al. Bevacizumab in combination with oxaliplatinbased chemotherapy as first-line therapy in metastatic colorectal cancer: a randomized phase III study. J Clin Oncol 2008; 26(12):2013-9.

7. Lièvre A, Bachet JB, Boige V, Cayre A, Le Corre D, Buc E, et al. KRAS Mutations as an independent prognostic factor in patients with advanced colorectal cancer treated with cetuximab. J Clin Oncol 2008;26(3):374-9.

8. Chung KY, Shia J, Kemeny NE, Shah M, Schwartz GK, Tse A, et al. Cetuximab shows activity in colorectal cancer patients with tumors that do not express the epidermal growth factor receptor by immunohistochemistry. J Clin Oncol 2005; 23(9):1803-10.

9. Sartore-Bianchi A, Moroni M, Veronese S, Carnaghi C, Bajetta E, Luppi G, et al. Epidermal growth factor receptor gene copy number and clinical outcome of metastatic colorectal cancer treated with panitumumab. J Clin Oncol 2007; 25(22):3238-45.

10. Janakiraman M, Vakiani E, Zeng Z, Pratilas CA, Taylor $\mathrm{BS}$, Chitale D, et al. Genomic and biological characterization of exon 4 KRAS mutations in human cancer. Cancer Res 2010; 70(14): 5901-11.

11. Vigil D, Cherfils J, Rossman KL, Der CJ. Ras superfamily
GEFs and GAPs: validated and tractable targets for cancer therapy? Nat Rev Cancer 2010; 10(12):842-57.

12. Smit VT, Boot AJ, Smits AM, Fleuren GJ, Cornelisse CJ, Bos JL. KRAS codon 12 mutations occur very frequently in pancreatic adenocarcinomas. Nucleic Acids Res 1988; 16(16):7773-82.

13. Karapetis CS, Khambata-Ford S, Jonker DJ, O'Callaghan $\mathrm{CJ}, \mathrm{Tu} \mathrm{D}$, Tebbutt NC, et al. K-ras mutations and benefit from cetuximab in advanced colorectal cancer. N Engl J Med 2008; 359(17):1757-65.

14. Amado RG, Wolf M, Peeters M, Van Cutsem E, Siena $\mathrm{S}$, Freeman DJ, et al. Wild-type KRAS is required for panitumumab efficacy in patients with metastatic colorectal cancer. J Clin Oncol 2008;26(10):1626-34.

15. Van Cutsem E, Köhne CH, Láng I, Folprecht G, Nowacki MP, Cascinu S, et al. Cetuximab plus irinotecan, fluorouracil, and leucovorin as first-line treatment for metastatic colorectal cancer: updated analysis of overall survival according to tumor KRAS and BRAF mutation status. J Clin Oncol 2011; 29(15):2011-9.

16. Neumann J, Zeindl-Eberhart E, Kirchner $T$, Jung A. Frequency and type of KRAS mutations in routine diagnostic analysis of metastatic colorectal cancer. Pathol Res Pract 2009; 205(12):858-62.

17. Bos JL. Ras oncogenes in human cancer: a review. Cancer Res 1989;49(17):4682-9.

18. Schubbert S, Shannon K, Bollag G. Hyperactive Ras in developmental disorders and cancer. Nat Rev Cancer 2007; 7(4):295-308.

19. Tan C, Du X. KRAS mutation testing in metastatic colorectal cancer. World J Gastroenterol 2012; 18(37):517180.

20. Vodolazhskiy DI, Kit OA, Gevorgyan YuA, Soldatkina NV. Features of KRAS mutation in advanced colorectal cancer. Eurasian J Oncol 2014; 3(3):82-4.

21. Phipps AI, Buchanan DD, Makar KW, Win AK, Baron $\mathrm{JA}$, Lindor NM, et al. KRAS-mutation status in relation to colorectal cancer survival: the joint impact of correlated tumour markers. Br J Cancer 2013; 108(8):1757-64. 